\title{
Schisandrin B synergizes docetaxel-induced restriction of growth and invasion of cervical cancer cells in vitro and in vivo
}

\author{
Chunmei Yan ${ }^{1 \#}$, Liya Gao ${ }^{2 \#}$, Xiaofei Qiu ${ }^{3}$, Chunxia Deng ${ }^{2 \wedge}$ \\ ${ }^{1}$ Department of Gynecology and Obstetrics, The Hospital of Lanzhou Jiao Tong University, Lanzhou, China; ${ }^{2}$ Department of Gynecology and \\ Obstetrics, Beijing Chuiyangliu Hospital, Beijing, China; ${ }^{3}$ Department of Gynecology and Obstetrics, The Central People’s Hospital of Tengzhou, \\ Tengzhou, China \\ Contributions: (I) Conception and design: C Deng; (II) Administrative support: C Deng; (III) Provision of study materials or patients: C Yan, L Gao; \\ (IV) Collection and assembly of data: X Qiu; (V) Data analysis and interpretation: C Yan, L Gao; (VI) Manuscript writing: All authors; (VII) Final \\ approval of manuscript: All authors. \\ "These authors contributed equally to this work. \\ Correspondence to: Chunxia Deng. Beijing Chuiyangliu Hospital, No.2, South Chui Yang Liu Street, Chaoyang District, Beijing, China. \\ Email: drzv60060@sina.cn.
}

Background: Cervical cancer is a prevalent tumor in women. Here we investigated the synergic effects of Schisandrin B (Sch B), an active compound extracted from the Chinese herb Schisandra Chinensis, in docetaxel (DTX)-induced restriction of growth and invasion of cervical cancer.

Methods: Caski cells were treated with Sch B and DTX for 24 hours. In vitro effects were investigated with Cell counting kit-8, western blotting, colony-forming, Transwell, Annexin V-FITC enabled flow cytometry. Then, in vivo experiments were engaged with Sch B $(20 \mathrm{mg} / \mathrm{kg})$ and DTX $(10 \mathrm{mg} / \mathrm{kg})$ for 30 days, and IHC were applied to validate the effects in vivo.

Results: Both Sch B and DTX reduced cell viability, inhibited colony formatting, induced apoptosis, and limited cell invasion. Co-administration of Sch B and DTX more significantly enhanced these changes. The relative levels of HPV infection and tumor progression related proteins $\mathrm{p}$-AKT/AKT, NF-kappaB, Cyclin D1, CDK-4, MMP-9, Notch1, $\beta$-catenin and p-p38/p38 were markedly inactivated. The effects of Sch B in cervical cancer were further confirmed in Caski cell-xenograft BALB/c nude mice. Co-administration of Sch B enhanced the anti-tumor effects of DTX in vivo, inhibited tumor formation, increased apoptotic cells, and reduced Ki67 and N-cadherin expression.

Conclusions: Altogether, Sch B enhanced the anti-tumor effects of DTX in vitro and in vivo via growth, invasion, and apoptosis regulating. The results supported therapies of co-administering Sch B and DTX to be developed in cervical cancer.

Keywords: Cervical cancer; Schisandrin B (Sch B); docetaxel (DTX); metastases

Submitted Jul 08, 2020. Accepted for publication Sep 19, 2020.

doi: 10.21037/atm-20-6109

View this article at: http://dx.doi.org/10.21037/atm-20-6109

\section{Introduction}

Cervical cancer is one of the most prevalent tumors in women, especially in impoverished areas (1). For about $90 \%$ of patients, cervical cancer is avoidable with the proactive treatment of human papillomavirus (HPV, the leading cause of cervical cancer) vaccination (2). However, since there remains a long way to eliminate poverty, cervical cancer could be a significant health issue in the upcoming decades (3). Consequently, the overall prognosis is still poor in women with metastatic or recurrent disease. HPV

\footnotetext{
^ ORCID: 0000-0003-0861-7008.
} 

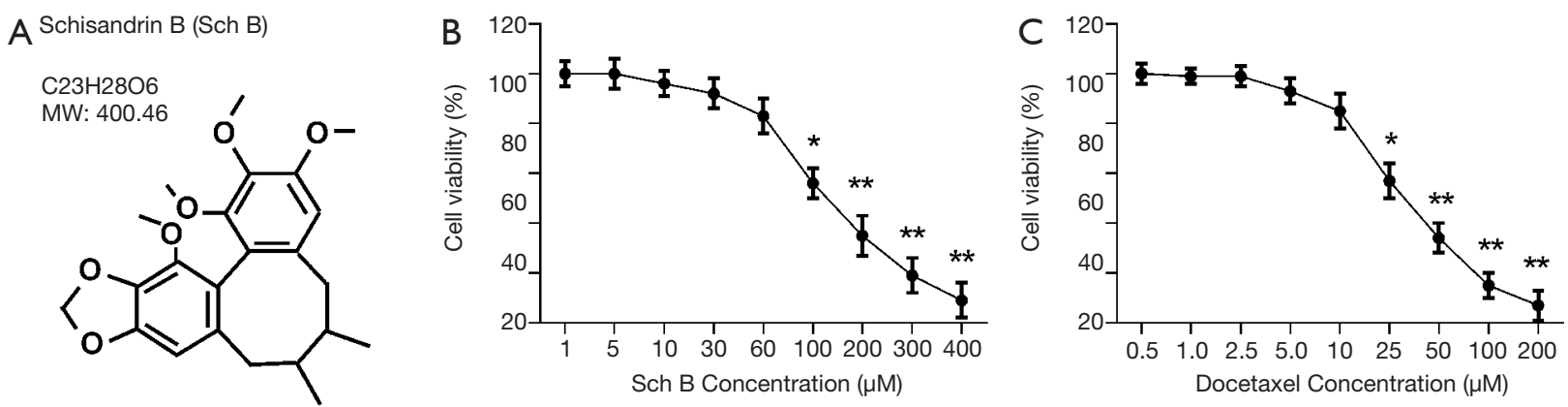

Figure 1 Viability of Caski cells treated with Sch B or DTX evaluated with CCK-8. Caski cells $\left(5 \times 10^{3}\right.$ in $\left.100 \mu \mathrm{L}\right)$ were treated with showed concentrations of Sch B or DTX for 24 hours. (A) The structure of Sch B. (B) viability of Caski cells treated with Sch B. (C) Viability of Caski cells treated with DTX. *, $\mathrm{P}<0.05$ and **, $\mathrm{P}<0.01$ versus control with one-way ANOVA from at least 3 replicates. Sch $\mathrm{B}$, Schisandrin $\mathrm{B}$; DTX, docetaxel; ANOVA, analysis of variance.

oncogenes including E5, E6 and E7 play pivotal roles in cervical cancer by activating tumor promotive pathways (AKT, Notch, Wnt, EGFR, Cyclins) and inhibiting tumor suppressor proteins (p53, PDZ). As a result, cell proliferation, migration and drug resistance were promoted, while cell apoptosis was reduced (4). The incorporation of the anti-VEGF agent and immunotherapeutic approaches have been able to extend overall survival (5). However, these therapeutics are still too expensive, especially for women in impoverished areas so far.

Docetaxel (DTX), a derivate of taxol, has been widely used in the treatment of several cancers. However, due to its poor water solubility, adverse effects inducing ingredients were used (6), which mostly limited its clinical use. Recently, DTX-loaded nanoparticles showed a potent anti-tumor effect in human breast cancer cells and human cervical cancer cells in vitro and in vivo (7), which shed light on the clinical application of DTX in cervical cancer.

Schisandrin (or Schizandrin, Sch, Figure 1A) are bioactive compounds extracted from Schisandra Chinensis, a standard Traditional Chinese Medicine. Sch derivatives, including Schisandrin B (Sch B), have been reported to target a series of signaling molecules and to have various pharmacological effects including anti-oxidant, anti-tumor, neuroprotection, myocardial preservation, and liver and kidney protection (8). The anti-tumor effects of Sch B were the results of inhibited cyclin and their relevant cyclindependent kinases, and induction of cell apoptosis. Sch B was predicted to dock Bax, Bcl-2, AKT, Cdk-2, Cdk-4, Cyclin D, Cyclin E, and MMP-9 with high affinity (8), which supported its anti-tumor activity. The potent organ protective and tumor-suppressive role of Sch B suggested that it could be an excellent adjuvant in tumor treatment. However, the clinical efficacy of Sch B is limited due to the poor solubility and low bioavailability (9). Indeed, functionalized doxorubicin plus Sch B co-delivery liposomes showed enhanced epithelial-mesenchymal transition (EMT) inhibition efficacy (10), and Sch B enhanced the anti-tumor activity of doxorubicin and reduced the cardiotoxicity in vivo, even without proper preparation (11). The earlier research and examples that strongly highlighted Sch B is a promising anti-tumor molecule, at least as an adjuvant.

In the present study, we attempted to characterize the effects of co-administered Sch B and DTX in cervical cancer. We briefly showed that Sch B enhanced the effects of DTX, including inhibited proliferation, induced apoptosis, and restrained invasion in Caski cells, and limited tumor progression in vivo.

We present the following article in accordance with the ARRIVE reporting checklist (available at http://dx.doi. org/10.21037/atm-20-6109).

\section{Methods}

\section{Animals}

The Hunan Science and Technology Department (SYXK 2019-0017) approved all procedures conducted on mice. All experiments were applied by Hunan SJA Laboratory Animal Co., Ltd. (Changsha, China) following the guide for the Care and Use of Laboratory Animals (National Research 119 Council, 1996). Four-week-old Balb/c female nude mice (bought from the Institute of Laboratory Animal Sciences, Chinese Academy of Medical Science) were used to evaluate 
the anti-tumor activity of Sch B and DTX. The mice were housed in a $12 / 12$ hour light/dark environment with a consistent temperature of $22 \pm 2{ }^{\circ} \mathrm{C}$ and humidity $55 \% \pm 5 \%$. After accustomed to a week, the mice were subcutaneously implanted with cell suspension holding $1 \times 10^{6}$ Caski cells (American Type Culture Collection, MA, USA) in the right flank. Then the mice were randomly divided into four groups ( $\mathrm{n}=5$ mice in each group). The mice were daily i.v. injected with saline, $20 \mathrm{mg} / \mathrm{kg}$ Sch B (PHL89786, Sigma-Aldrich, St. Louis, MO, USA), $10 \mathrm{mg} / \mathrm{kg}$ DTX (Medchemexpress, Shanghai, China), or $20 \mathrm{mg} / \mathrm{kg}$ Sch B $+10 \mathrm{mg} / \mathrm{kg}$ DTX for 30 days. The tumor volume $(\mathrm{V})$ is evaluated every five days by measuring the length $(\mathrm{L})$ and width $(W)$ and calculated using the following formula: $\mathrm{V}=\mathrm{L} \times \mathrm{W}^{2} \times 0.52, \mathrm{~W}<\mathrm{L}$. Finally, the mice were sacrificed by $\mathrm{CO}_{2}$ inhalation, and the tumors were photographed and fixed with 4\% Paraformaldehyde Fix Solution (Beyotime Biotechnology, Shanghai, China) for later experiments.

\section{Cell culture and viability}

The Caski cells were supported in RPMI 1640 medium supplemented with $10 \%$ FBS, 100 units/mL penicillin, and $100 \mathrm{mg} / \mathrm{mL}$ streptomycin (purchased from Hyclone, Logan, UT, USA). The cells were cultured in a humidified incubator with $5 \% \mathrm{CO}_{2}$ at $37^{\circ} \mathrm{C}$. The CCK-8 kit (Dojindo, Shanghai, China) was used to investigate cell viability. Each well of 96 -well plates was seeded with $5 \times 10^{3}$ Caski cells in the $100 \mu \mathrm{L}$ medium in the logarithmic growth phase and cultured at $37{ }^{\circ} \mathrm{C}$ and $5 \% \mathrm{CO}_{2}$. After culturing for 24 hours, cells were treated for a further 24 hours with

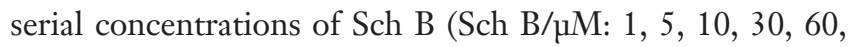
100, 200, 300, 400), DTX (Doc/nM: 0.5, 1, 2.5, 5, 10, 25, $50,100,200)$, vehicle control (DMSO) or blank control in triplicate. Cisplatin $(50 \mu \mathrm{M}$, Sigma-Aldrich) was used as positive control. Two hours before the termination of each time point, $10 \mu \mathrm{L}$ CCK-8 was added to each well. The optical density (OD) of each well was measured with a microplate reader (Bio-Rad Laboratories, Inc., Berkeley, CA, USA) at the wavelength of $450 \mathrm{~nm}$. The inhibition curves of Sch B and DTX were achieved.

\section{Colony formation}

The Caski cells were trypsinized and dispensed into individual wells of 6-well culture dishes with a density of 200 cells per well. The cells were treated for ten days (saline, $10 \mathrm{nM}$ DTX, $60 \mu \mathrm{M}$ Sch B or $10 \mathrm{nM}$ DTX + $60 \mu \mathrm{M}$ Sch B.
The concentrations were used for later iv vitro experiments) without changing the medium. Paraformaldehyde Fix Solution (4\%) was used to fix cell colonies for 10 minutes, and $0.5 \%$ crystal violet (Beyotime) was employed to stain cell colonies for 5 minutes. After that, colony formation rates were measured. The experiment was performed in triplicate.

\section{Flow cytometry}

Annexin V-fluorescein isothiocyanate (FITC) Apoptosis Detection Kit (556547, BD Biosciences, San Diego, CA, USA) was used to detect apoptotic Caski cells. Briefly, $1 \times 10^{6}$ cells per well were seeded in 6-well plates overnight and then exposed to treatments for 24 hours. Then, trypsinization collected the cells, washed twice with cold phosphate-buffered saline (PBS), and resuspended in a $500 \mathrm{~mL} 1 \times$ binding buffer. The cells were further mixed with $5 \mathrm{~mL}$ annexin V-FITC and $5 \mathrm{~mL}$ propidium iodide (PI) and incubated for 15 minutes in the dark at room temperature. Finally, the apoptotic cells are analyzed with flow cytometry (BD Bioscience).

\section{Western blotting}

The treated Caski cells were lysed in RadioImmunoprecipitation Assay (RIPA) Lysis Buffer (Beyotime) and collected. After balancing with a Bicinchoninic Acid (BCA) Protein Assay Kit (Beyotime), the lysates were mixed with $5 \times$ Loading Buffer (Beyotime), fractionated on 10\% sodium dodecyl sulfate-polyacrylamide gel electrophoresis (SDS-PAGE) gels (Beyotime), and transferred to $0.2 \mu \mathrm{m}$ polyvinylidene difluoride membranes (Millipore, Billerica, MA, USA). The membranes were blocked with $5 \%$ milk in Tris-buffered saline with $0.1 \%$ Tween 20 (TBST) and incubated overnight at $4{ }^{\circ} \mathrm{C}$ in primary antibodies (Abcam, Cambridge, UK). After washing with TBST, the membranes were incubated with secondary antibodies for 1 hour at room temperature. Protein bands were detected using enhanced chemiluminescence (Fude Biological Technology). $\beta$-Actin was used for normalization of protein loading. Antibodies used were: anti-Ki67 (ab92742), anti-caspase 3 (ab32351), anti-Bax (ab32503), anti-Bcl-2 (ab59348), antiVEGF (ab150375), anti-E-cadherin (ab40772), anti-Ncadherin (ab76011), anti-Vimentin (ab92547), anti-AKT (ab8805), anti-p-AKT (phospho T308, ab38449), anti-NFкB P65 (ab207297), anti-EGFR (ab52894), anti-Cyclin D1 (134175), anti-CDK-4 (ab 108357), anti-MMP-9 (ab38898), anti-Notch1 (ab52627), anti- $\beta$-catenin (ab68183), anti-p53 
(ab32389), anti-p38 (ab170099), anti-p-p38 (ab195049), anti- $\beta$-actin (ab8227) and secondary antibody (ab205718).

\section{Transwell}

The Transwell assay was performed to assess the invasion capability of treating Caski cells as previously reported (12). The 24-well BioCoat cell culture inserts (BD Biosciences, Bedford, MA, USA) with a polyethylene terephthalate membrane ( $8-\mu \mathrm{m}$ porosity) is used. The upper chamber membranes were coated with $100 \mu \mathrm{L}$ Matrigel $(50 \mu \mathrm{g} / \mathrm{mL}$, BD Biosciences, San Jose, CA, USA) and then incubated for $6 \mathrm{~h}$ at $37^{\circ} \mathrm{C}$. About $1 \times 10^{5}$ Caski cells were added into the upper chamber in the serum-free medium, and $0.6 \mathrm{~mL}$ complete culture medium was added into the lower chamber. Both the upper and lower chambers were added by showing drugs for treatment. After incubation for $24 \mathrm{~h}$, the cells were washed twice with PBS, the remaining cells in the upper chamber were wiped away using cotton swabs. After fixing with 4\% Paraformaldehyde Fix Solution, 0.1\% crystal violet was used to stain. The number of invasion cells on the bottom surface of the membrane was recorded by the imaging device and counted under a light microscope.

\section{Tissue staining}

The fixed tissues are paraffin-embedded to generate $5 \mu \mathrm{m}$ sections. The sections were deparaffinized with xylene, rehydrated in an alcohol gradient, immersed in $3 \% \mathrm{H}_{2} \mathrm{O}_{2}$. For TdT-mediated dUTP Nick-End Labeling (TUNEL, Beyotime), sections were stained with Biotin-labeling solution and Streptavidin-HRP work solution and evaluated according to instructions. For immunohistochemistry, sections were incubated with primary antibodies for Ki67 $(1: 1,000$, Abcam $)$ and $\mathrm{N}$-cadherin $(1: 500$, Abcam $)$ at $4{ }^{\circ} \mathrm{C}$ overnight. The processed sections were incubated with a secondary antibody using the ABC kit (Vector Laboratories. Burlingame, CA, USA) for $1 \mathrm{~h}$ at room temperature. The sections were visualized by diaminobenzidine reaction and counterstaining with hematoxylin. The number of Ki67 and EGFR positive cells and apoptotic cells is analyzed from 3 high-power random fields of each slide. Sections with the absence of primary antibody and the same concentration of secondary antibody served as a negative control.

\section{Statistics}

Statistical analysis was performed using GraphPad Prism
7.0, and the bar graphs were shown as mean \pm standard deviation (SD). Statistical comparison among groups was performed with a $t$-test or one-way analysis of variance (ANOVA). $\mathrm{P}<0.05$ was considered statistical significance.

\section{Results}

\section{Sch B and DTX reduced the viability of Caski calls}

The viability of Caski cells treated with Sch B or DTX was investigated. As shown in Figure 1B,C, both Sch B and DTX dose-dependently reduce the viability of Caski cells. Then, $60 \mu \mathrm{M}$ of Sch B and $10 \mathrm{nM}$ of DTX was routinely used in further experiments to elucidate their underlined effects and mechanisms.

\section{Sch B and DTX inbibited proliferation and induced apoptosis of Caski cells}

Proliferation and apoptosis are the most targeted processes for anticancer drugs. The effects of Sch B and DTX in Caski cells were then evaluated. The result showed that DTX significantly inhibited proliferation and of Caski cells, which is enhanced with Sch B (Figure 2A). Apoptosis is markedly induced in both Sch B, and DTX treated cells, and more significantly increased in Sch B + DTX treated cells (Figure 2B). The inhibited proliferation and induced apoptosis were further confirmed with reduced protein expression of $\mathrm{Ki67}$, and increased ratio of Cleaved caspase 3/caspase 3 and Bax/Bcl-2, respectively (Figure 2C).

\section{Sch B and DTX limited invasion of Caski cells}

Next, the effects of Sch B and DTX on the invasion of Caski cells were investigated. Both Sch B and DTX reduced cells passing through the Matrigel, and Sch B + DTX more significantly reduced invasive cells (Figure $3 A$ ). The inhibited invasion was further confirmed with reduced protein expression of VEGF, N-cadherin, and Vimentin, and increased protein level of E-cadherin (Figure 3B).

\section{Sch B and DTX have synergistic effects in many ways}

The possible primary regulators that could be involved were further investigated (Figure 4A). AKT, NF-kappaB, Cyclin D1 and CDK-4 were significantly inhibited with either Sch B or DTX, but EGFR was not significantly inhibited with Sch B and DTX treatment (Figure 4B). MMP-9, 

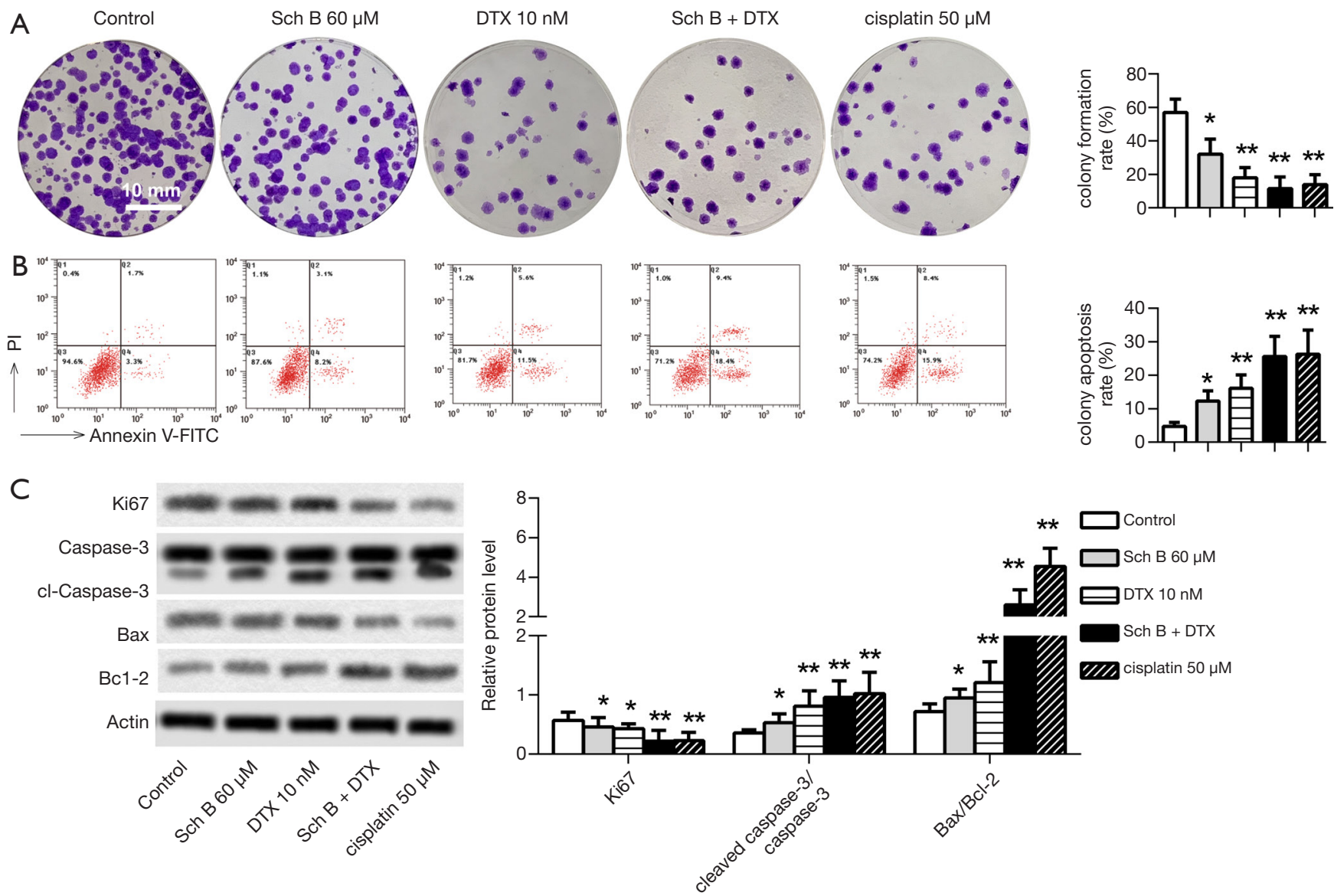

Figure 2 The inhibited proliferation and induced apoptosis with Sch B and DTX. (A) Colony formation of 200 Caski cells in 6-well plates treated with showed drugs for ten days and stained with crystal violet. (B) Apoptosis of Caski cells treated with showing drugs for 24 hours evaluated with flow cytometry. (C) Protein expression of Ki67, (Cleaved) Caspase-3, Bax, and Bcl-2. *, $\mathrm{P}<0.05$ and **, $\mathrm{P}<0.01$ versus control with $t$-test from at least 3 replicates. Sch B, Schisandrin B; DTX, docetaxel.

Notch1 and $\beta$-catenin were inactivated with DTX and more markedly inhibited in Sch B + DTX group. Relative level of p53 was induced while p-p38/p38 was inhibited with Sch B, and DTX treatment seemed to have no effect on both $\mathrm{p} 53$ and p38 levels (Figure 4C). The results implied the complex synergistic effects in the anti-tumor effects of Sch B and DTX.

\section{Sch B enhanced the anti-tumor effects of DTX in vivo}

Finally, the effects of Sch B and DTX in vivo were evaluated in Caski xenograft mice. After administering for 30 days, tumor formation in all treated mice was significantly smaller than control, and co-administration of Sch B and DTX showed a marked inhibition of tumor growth (Figure $5 A$ ). A similar trend was observed in TUNEL stained sections from the tumors, with Sch B + DTX significantly induced apoptotic cells in xenograft tumors (Figure 5B,C). Apoptosis in the tumor tissues were further validated with relative expression of cleaved Caspase-3/Caspase-3 and Bax/Bcl-2 (Figure 5D). What is more, the expression of Ki67 and $\mathrm{N}$-cadherin was significantly reduced in Sch B + DTX treated tissues (Figure $5 B, E, F$ ). The results supported the effects of Sch B and DTX in cervical cancer in vivo.

\section{Discussion}

It was estimated that 569,847 new cases and 311,365 deaths occurred globally in cervical cancer in 2018 (13). Prevention of cervical cancer with vaccination could avoid risks from common HPV subtypes, with which a one-third reduction in the rate of premature 

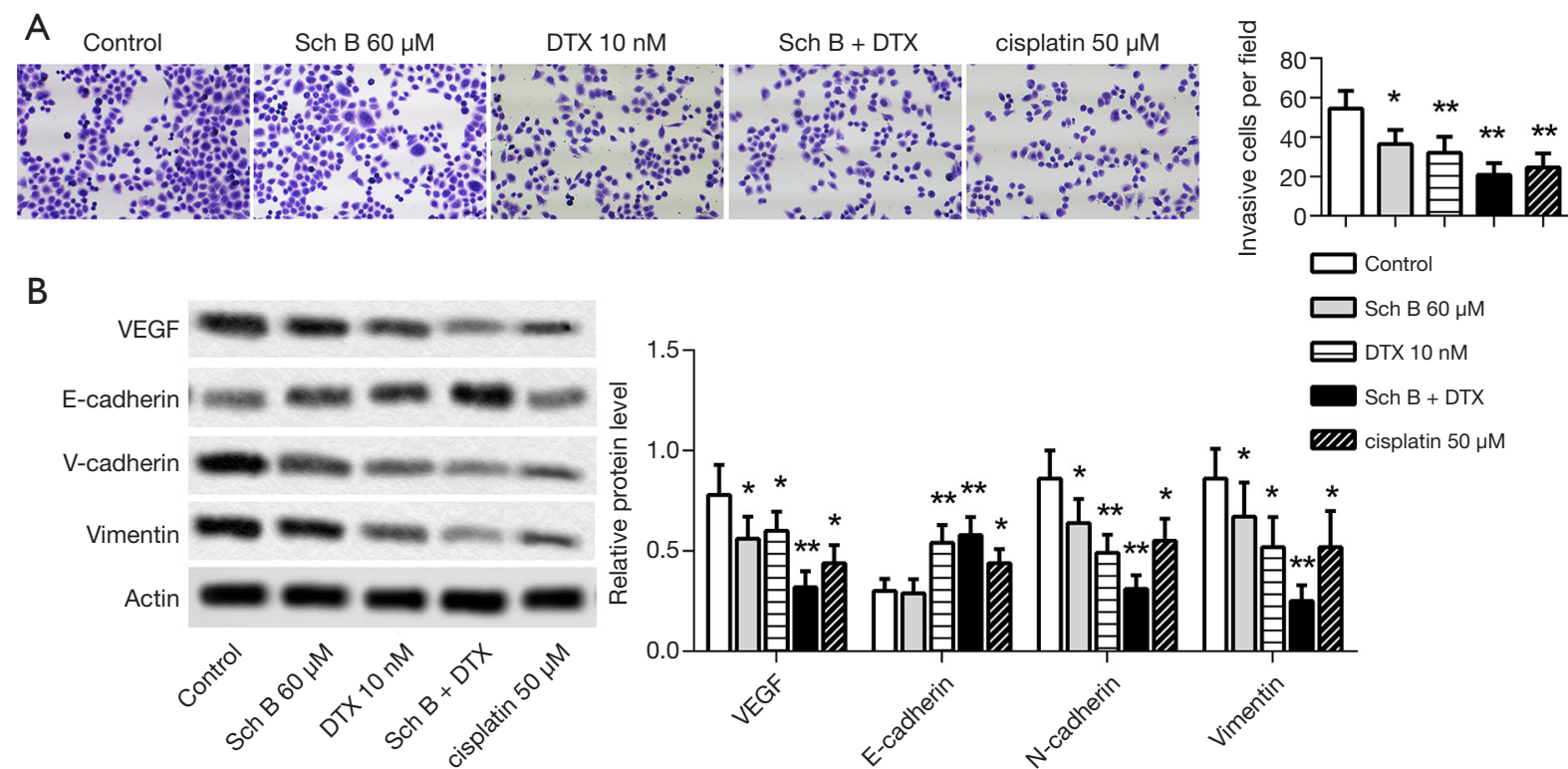

Figure 3 Sch B and DTX inhibited the invasion of Caski cells. The Caski cells were treated with showing drugs for 24 hours. (A) The invasive cells showed with Transwell stained with crystal violet and observed under 200× scope. (B) The relative protein expression of VEGF, E-cadherin, N-cadherin, and Vimentin was investigated. *, $\mathrm{P}<0.05$ and ${ }^{* *}, \mathrm{P}<0.01$ versus control with $t$-test from at least 3 replicates. Sch $\mathrm{B}$, Schisandrin B; DTX, docetaxel.
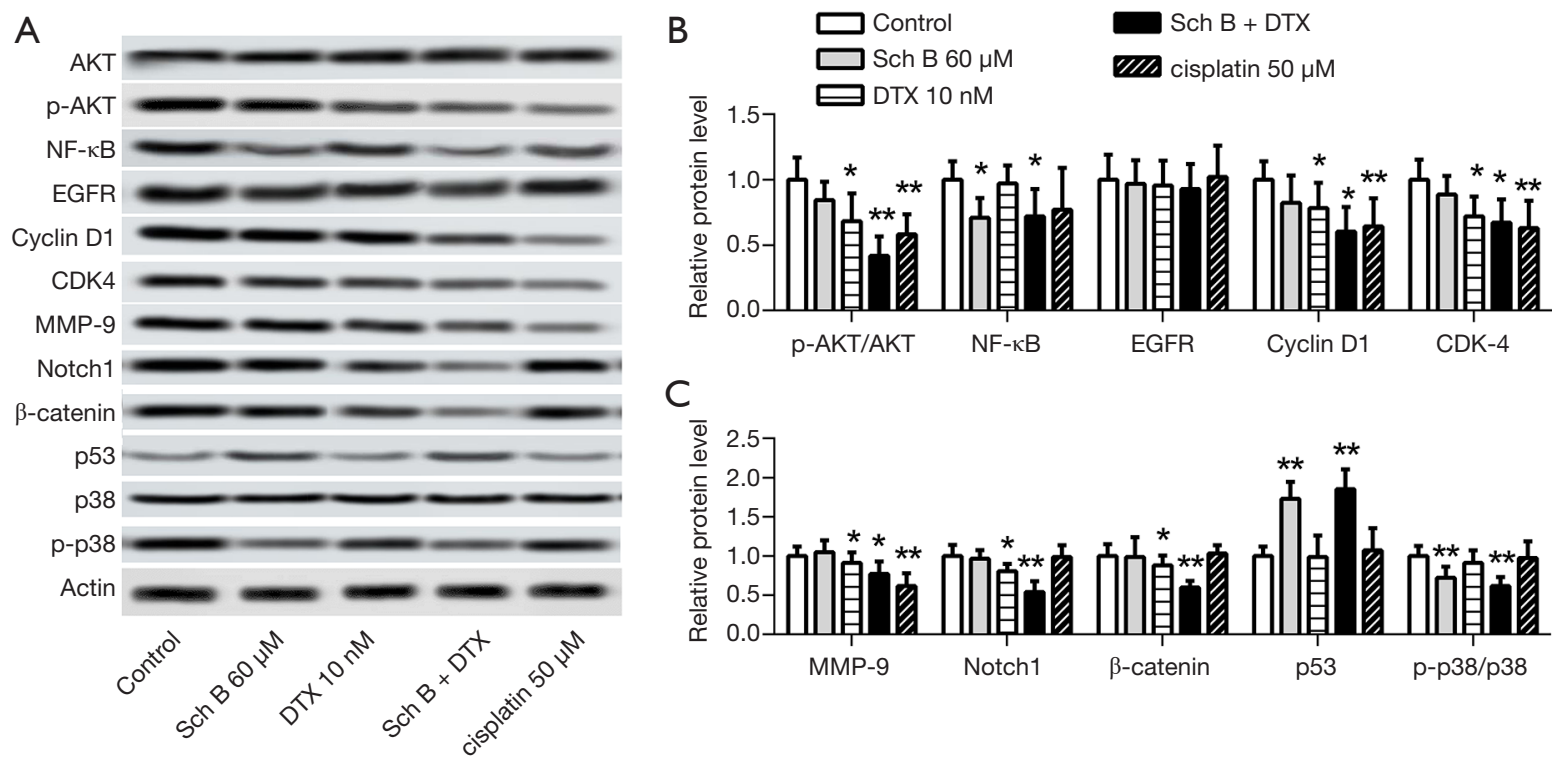

Figure 4 Sch B and DTX have complex synergistic effects. The Caski cells were treated with saline, $10 \mathrm{nM}$ DTX, $60 \mu \mathrm{M}$ Sch B, or $10 \mathrm{nM}$ DTX $+60 \mu \mathrm{M}$ Sch B for 24 hours. (A) Expression of HPV infection and Sch B treatment related proteins were characterized. (B) The relative protein expression of AKT, NF-kappaB, EGFR, Cyclin D1 and CDK-4 was shown. (C) The relative protein expression of MMP-9, Notch1, $\beta$-catenin, p53 and p-p38/p38 was characterized. *, $\mathrm{P}<0.05$ and ${ }^{* *}, \mathrm{P}<0.01$ versus control with $t$-test from at least 3 replicates. Sch $\mathrm{B}$, Schisandrin B; DTX, docetaxel. 

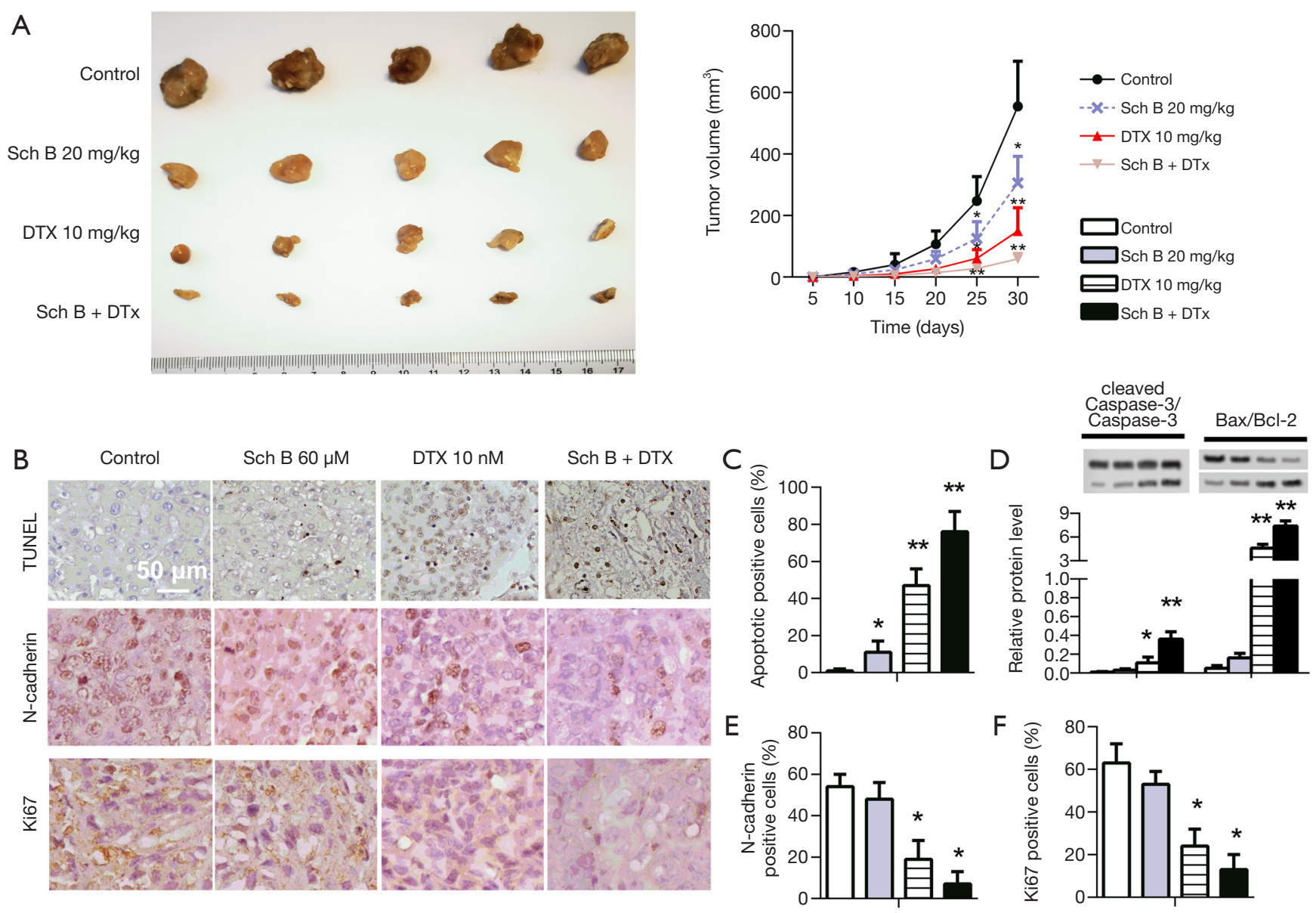

Figure 5 The effects of Sch B and DTX in vivo. Twenty Balb/c female nude mice were seeded with $1 \times 10^{6}$ Caski cells and daily i.v. injected with showing drugs for 30 days. The tumor volume was recorded every five days. (A) The tumor volumes recorded and harvested on day 30 . (B) Staining of the xenograft tumors observed under 400x scope. The Tunnel staining was analyzed with Image-Pro Plus (C). (D) Apoptosis in tumor tissues was indicated with relative levels of cleaved Caspase-3/Caspase-3 and Bax/Bcl-2. N-cadherin (E), and Ki67 (F) positive staining cells being counted. *, $\mathrm{P}<0.05$ and **, $\mathrm{P}<0.01$ versus control with $t$-test or one-way ANOVA from 5 replicates. Sch B, Schisandrin B; DTX, docetaxel; ANOVA, analysis of variance.

mortality from cervical cancer in lower-middle-income countries within ten years (3). For women with cervical cancer, effective treatments were still lacking, especially for late-stage patients. Adjuvant treatment of cervical cancer with cisplatin and gemcitabine is possibly inferior to chemoradiation therapy alone in locally advanced cervical cancer patients (median age 48 years) (14). However, the prognosis was poorer for younger age women who received radiation-based treatment (15). In the present research, we show that co-administration of Sch B and DTX inhibited proliferation and invasion, and induced apoptosis of Caski cells both in vivo and in vitro, supporting their therapeutic value in cervical cancer.
Sch derivatives are broadly implicated in suppressing various tumors, including cervical cancer. Used alone or as an adjuvant, Sch derivatives possibly have multiple beneficial effects to cancer patients, including immunoregulation (16), cycle arrest and apoptosis induction (17), and inhibition of proliferation, migration, and invasion (18). Importantly, Sch derivatives target multiple tumorpromoting signaling pathways to enhance the effects of other anti-tumor drugs, including gefitinib and DTX $(19,20)$. Using these pathways could be an excellent strategy to develop novel therapies for tumors to overcome drug resistance. For Sch B, the anti-tumor effects were realized with a series of cell cycle and apoptosis regulating proteins, 
including Bax, Bcl-2, P53, AKT, NF-kappaB, cyclin D1, cyclin-dependent kinases, MMP-9, Notch, wnt/ $\beta$-catenin and VEGF, most of which were tumor-promoting and HPV infection related molecules (8). Using Sch B in cancer therapy could possibly avoid bypass activation of tumor promotive processes, which was strongly supported by current studies. In the present research, we show that Sch $B$ enhanced the effects of DTX, with inactivated AKT, NF-kappaB, Cyclin D1, CDK-4, MMP-9, Notch1, p38 and $\beta$-catenin as well as activated $\mathrm{p} 53$. The suppressed tumor progression was further confirmed in Caski cell Xenograft mice. The results supported the complex mechanism of action for Sch B to enhance the effects of DTX.

DTX is not routinely used in cervical cancer, as the distribution is limited. Strikingly, DTX has been implicated to have promising therapeutic effects in vivo and even in clinical studies when co-administered or with improved formulation (21-23). Taxanes, including DTX, targets tubulin to arrest the cell cycle, a reduced tubulin polymer associated with resistance of Taxanes (24). Other possible DTX effects and resistance-related proteins also include multidrug resistance protein 1 (25), AKT (26), STAT3 (27), NF-kappaB, and MMPs $(28,29)$, which was implicated to be targeted with Sch B (8). The implicated proteins were briefly investigated in the current research. Consequently, Sch B enhanced the inhibition of AKT, Cyclin D1, CDK4 and MMP-9, Notch1, p38, $\beta$-catenin and suppressed NF-kappaB in DTX treated Caski cells. The enhanced anti-tumor effect of Sch B was related to the strengthened suppression of both tumor-promoting molecules and DTX elicited bypass processes.

The inactivation of p-AKT/AKT, NF-кB, VEGF, Cyclin D1, CDK-4, MMP-9, Notch-1, $\beta$-catenin and p-p38/p38 have important implications especially in cervical cancers. As most of these molecules are involved in both HPV infection and tumor progression $(4,8)$, administration of Sch B could possibly have effects on both $\mathrm{PHV}$ virus and cervical cancer cells. EGFR was not changed possibly because the low dosage. Similarly, Caski cells were not strikingly inhibited with $60 \mu \mathrm{M}$ of Sch B, and so were changes in many of the proteins mentioned above. However, $60 \mu \mathrm{M}$ in vitro or $20 \mathrm{mg} / \mathrm{kg}$ in vivo of Sch B was enough to be an adjavant for DTX. Our results strongly supported the possible application of Sch B in cervical cancer treatment.

There are limitations to this paper. Other potentially beneficial effects of Sch B as an adjuvant for DTX were not explored. Sch B suppressed injury and fibrosis of the kidney, liver, and lung (30-32), which possibly reduce the adverse effects of DTX. However, there are possibilities that part of the function of Sch B could be harmful to cancer therapy. The involvement of AKT, NF-kappaB, Cyclin D1, CDK-4, and MMP-9 were not investigated in-depth, and many other targets reported to be targets of Sch B or DTX were not tested. All these problems need to be further studied.

In conclusion, we showed that Sch B was a promising adjuvant for DTX in cervical cancer. The inhibited proliferation and EMT, and inhibited apoptosis was enhanced with Sch B, which could be related to the inactivation of AKT, NF-kappaB, Cyclin D1, CDK-4, and MMP-9.

\section{Acknowledgments}

Funding: None.

\section{Footnote}

Reporting Checklist: The authors have completed the ARRIVE reporting checklist. Available at http://dx.doi. org/10.21037/atm-20-6109

Data Sharing Statement: Available at http://dx.doi. org/10.21037/atm-20-6109

Conflicts of Interest: All authors have completed the ICMJE uniform disclosure form (available at http://dx.doi. org/10.21037/atm-20-6109). The authors have no conflicts of interest to declare.

Ethical Statement: The authors are accountable for all aspects of the work in ensuring that questions related to the accuracy or integrity of any part of the work are appropriately investigated and resolved. The Hunan Science and Technology Department approved all procedures conducted on mice (SYXK 2019-0017). The experiments were allpied following the guide for the Care and Use of Laboratory Animals (National Research 119 Council, 1996).

Open Access Statement: This is an Open Access article distributed in accordance with the Creative Commons Attribution-NonCommercial-NoDerivs 4.0 International License (CC BY-NC-ND 4.0), which permits the noncommercial replication and distribution of the article with the strict proviso that no changes or edits are made and the original work is properly cited (including links to both the formal publication through the relevant DOI and the license). 
See: https://creativecommons.org/licenses/by-nc-nd/4.0/.

\section{References}

1. Siegel RL, Miller KD, Jemal A. Cancer statistics, 2019. CA Cancer J Clin 2019;69:7-34.

2. Cleveland AA, Gargano JW, Park IU, et al. Cervical adenocarcinoma in situ: Human papillomavirus types and incidence trends in five states, 2008-2015. Int J Cancer 2020;146:810-8.

3. Canfell K, Kim JJ, Brisson M, et al. Mortality impact of achieving WHO cervical cancer elimination targets: a comparative modelling analysis in 78 low-income and lower-middle-income countries. Lancet 2020;395:591-603.

4. Chen J. Signaling pathways in HPV-associated cancers and therapeutic implications. Rev Med Virol 2015:25 Suppl 1:24-53.

5. Geng F, Fan MJ, Li J, et al. Knockdown of METTL14 inhibits the growth and invasion of cervical cancer. Transl Cancer Res 2019;8:2307-15.

6. Pu J, Qin SS, Ding JX, et al. A randomized controlled study of single-agent cisplatin and radiotherapy versus docetaxel/cisplatin and radiotherapy in high-risk earlystage cervical cancer after radical surgery. J Cancer Res Clin Oncol 2013;139:703-8.

7. Wang Y, Zuo A, Huang X, et al. Docetaxel-loaded PAMAM-based poly ( $\gamma$-benzyl-l-glutamate)-b-d$\alpha$-tocopheryl polyethylene glycol 1000 succinate nanoparticles in human breast cancer and human cervical cancer therapy. J Microencapsul 2019;36:552-65.

8. Nasser MI, Zhu S, Chen C, et al. A Comprehensive Review on Schisandrin B and Its Biological Properties. Oxid Med Cell Longev 2020;2020:2172740.

9. Shao M, Yang W, Han G. Protective effects on myocardial infarction model: delivery of schisandrin B using matrix metalloproteinase-sensitive peptide-modified, PEGylated lipid nanoparticles. Int J Nanomedicine 2017;12:7121-30.

10. Cai FY, Yao XM, Jing M, et al. Enhanced antitumour efficacy of functionalized doxorubicin plus schisandrin B co-delivery liposomes via inhibiting epithelialmesenchymal transition. J Liposome Res 2020:1-17.

11. Xu Y, Liu Z, Sun J, et al. Schisandrin B prevents doxorubicin-induced chronic cardiotoxicity and enhances its anticancer activity in vivo. PLoS One 2011;6:e28335.

12. Lu X, Yang F, Chen D, et al. Quercetin reverses docetaxel resistance in prostate cancer via androgen receptor and PI3K/Akt signaling pathways. Int J Biol Sci 2020;16:1121-34.
13. Bray F, Ferlay J, Soerjomataram I, et al. Global cancer statistics 2018: GLOBOCAN estimates of incidence and mortality worldwide for 36 cancers in 185 countries. CA Cancer J Clin 2018;68:394-424.

14. da Costa SCS, Bonadio RC, Gabrielli FCG, et al. Neoadjuvant Chemotherapy With Cisplatin and Gemcitabine Followed by Chemoradiation Versus Chemoradiation for Locally Advanced Cervical Cancer: A Randomized Phase II Trial. J Clin Oncol 2019;37:3124-31.

15. Yagi A, Ueda Y, Kakuda M, et al. Epidemiologic and Clinical Analysis of Cervical Cancer Using Data from the Population-Based Osaka Cancer Registry. Cancer Res 2019;79:1252-9.

16. Lin RD, Mao YW, Leu SJ, et al. The immuno-regulatory effects of Schisandra chinensis and its constituents on human monocytic leukemia cells. Molecules 2011;16:4836-49.

17. Lv XJ, Zhao LJ, Hao YQ, et al. Schisandrin B inhibits the proliferation of human lung adenocarcinoma A549 cells by inducing cycle arrest and apoptosis. Int J Clin Exp Med 2015;8:6926-36.

18. Ding Q, Li X, Sun Y, et al. Schizandrin A inhibits proliferation, migration and invasion of thyroid cancer cell line TPC- 1 by down regulation of microRNA-429. Cancer Biomark 2019;24:497-508.

19. Xian H, Feng W, Zhang J. Schizandrin A enhances the efficacy of gefitinib by suppressing IKK /NF- B signaling in non-small cell lung cancer. Eur J Pharmacol 2019;855:10-9.

20. Su X, Gao C, Shi F, et al. A microemulsion co-loaded with Schizandrin A-docetaxel enhances esophageal carcinoma treatment through overcoming multidrug resistance. Drug Deliv 2017;24:10-9.

21. Chen P, Jiao L, Wang DB. Squamous cell carcinoma antigen expression in tumor cells is associated with the chemosensitivity and survival of patients with cervical cancer receiving docetaxel-carboplatin-based neoadjuvant chemotherapy. Oncol Lett 2017;13:1235-41.

22. Xu G, Yu X, Zhang J, et al. Robust aptamer-polydopaminefunctionalized M-PLGA-TPGS nanoparticles for targeted delivery of docetaxel and enhanced cervical cancer therapy. Int J Nanomedicine 2016;11:2953-65.

23. Huang X, Lan C, Huang H, et al. Neoadjuvant docetaxel combined with cisplatin and followed by radical surgery for the treatment of locally advanced (stage IB2 - IIB) cervical cancer: preliminary results of a single-institution experience. Expert Opin Pharmacother 2011;12:165-73.

24. Duran GE, Wang YC, Moisan F, et al. Decreased levels 
of baseline and drug-induced tubulin polymerisation are hallmarks of resistance to taxanes in ovarian cancer cells and are associated with epithelial-to-mesenchymal transition. Br J Cancer 2017;116:1318-28.

25. Yan M, Wang J, Ren Y, et al. Over-expression of FSIP1 promotes breast cancer progression and confers resistance to docetaxel via MRP1 stabilization. Cell Death Dis 2019;10:204.

26. Zhang X, Shao J, Li X, et al. Docetaxel promotes cell apoptosis and decreases SOX2 expression in CD133-expressing hepatocellular carcinoma stem cells by suppressing the PI3K/AKT signaling pathway. Oncol Rep 2019;41:1067-74.

27. Hu F, Zhao Y, Yu Y, et al. Docetaxel-mediated autophagy promotes chemoresistance in castration-resistant prostate cancer cells by inhibiting STAT3. Cancer Lett 2018;416:24-30.

28. Kim SM, Lee SY, Yuk DY, et al. Inhibition of NF-kappaB by ginsenoside $\mathrm{Rg} 3$ enhances the susceptibility of colon

Cite this article as: Yan C, Gao L, Qiu X, Deng C. Schisandrin $\mathrm{B}$ synergizes docetaxel-induced restriction of growth and invasion of cervical cancer cells in vitro and in vivo. Ann Transl Med 2020;8(18):1157. doi: 10.21037/atm-20-6109 cancer cells to docetaxel. Arch Pharm Res 2009;32:755-65.

29. Vacca A, Ribatti D, Iurlaro M, et al. Docetaxel versus paclitaxel for antiangiogenesis. J Hematother Stem Cell Res 2002;11:103-18.

30. Cao G, Li S, Shi H, et al. Schisandrin B attenuates renal fibrosis via miR-30e-mediated inhibition of EMT. Toxicol Appl Pharmacol 2019;385:114769.

31. Cuiqiong W, Chao X, Xinling F, et al. Schisandrin B suppresses liver fibrosis in rats by targeting miR-101$5 \mathrm{p}$ through the TGF- $\beta$ signaling pathway. Artif Cells Nanomed Biotechnol 2020;48:473-8.

32. Zhang D, Liu B, Cao B, et al. Synergistic protection of Schizandrin B and Glycyrrhizic acid against bleomycininduced pulmonary fibrosis by inhibiting TGF- $\beta 1 /$ Smad2 pathways and overexpression of NOX4. Int Immunopharmacol 2017;48:67-75.

(English Language Editor: J. Chapnick) 